\title{
Density Profiles in Open Superdiffusive Systems
}

\author{
Stefano Lepri ${ }^{1}$ and Antonio Politi ${ }^{1}$ \\ ${ }^{1}$ Consiglio Nazionale delle Ricerche, Istituto dei Sistemi Complessi, \\ via Madonna del Piano 10, I-50019 Sesto Fiorentino, Italy
}

(Dated: May 25, 2022)

\begin{abstract}
We numerically solve a discretized model of Lévy random walks on a finite one-dimensional domain in the presence of sources and with a reflection coefficient $r$. At the domain boundaries, the steady-state density profile is non-analytic. The meniscus exponent $\mu$, introduced to characterize this singular behavior, uniquely identifies the whole profile. Numerical data suggest that $\mu=$ $\alpha / 2+r(\alpha / 2-1)$, where $\alpha$ is the Lévy exponent of the step-length distribution. As an application, we show that this model reproduces the temperature profiles obtained for a chain of oscillators displaying anomalous heat conduction. Remarkably, the case of free-boundary conditions in the chain correspond to a Lévy walk with negative reflection coefficient.
\end{abstract}

PACS numbers: 05.60.-k 05.40.Fb 44.10.+i

Anomalous diffusion and transport are ubiquitous phenomena that occur in many complex systems, ranging from molecular motion to human mobility [1]. In the framework of random walk theory much progress has been made in the last years in the characterization of superdiffusive motion in infinite domains [1, 2]. Extension to the case of finite systems is relatively less developed [3, 4]. Typically, only the simple case of absorbing Boundary Conditions (BC) without sources has been treated [5]. An even less studied problem is that of $f_{i}$ nite and open superdiffusive systems where particles and energy are steadily exchanged with some external reservoirs [6]. This setup describes a variety of systems with anomalous transport in the off-equilibrium regime. For many-particle systems this is exemplified by anomalous heat conduction in low-dimensional lattices 7, 8], as well as by enhanced thermodiffusion effects [9]. Moreover, there exist at least two physical setups where the theoretical predictions can be experimentally tested: light superdiffusion [10,11] and anomalous heat conduction in individual nanotubes [12].

In the present Letter we study the spatial distribution of the relevant field (particle density, temperature, light intensity, depending on the context) in the presence of an external gradient. By numerically investigating a model of particles performing Lévy flights or walks, we find that the shape of the profile is nonlinear in the thermodynamic/continuum limit. This is due to the long-range correlations which affect the transport far away from the boundaries. A comparison with the temperature profiles obtained in a model of heat conductivity indicates that the shape is fully determined by the exponent of the underlying Lévy process and by the BC. However, the connection between the (free/fixed) $\mathrm{BC}$ of the conduction problem and those of the diffusive process (reflectivity) is not trivial. We indeed find that free $\mathrm{BC}$ correspond to a negative reflectivity.

The model - Let $i$ denote the position of a discrete-time random walker on a finite one-dimensional lattice $(1 \leq$ $i \leq N)$. In between consecutive scattering events, the particle either jumps instantaneously (Lévy flight - LF) or moves with unit velocity (Lévy walk - LW [13]) over a distance of $m$ sites, that is randomly selected according to the step-length distribution

$$
\psi_{m}=\frac{q}{|m|^{1+\alpha}}, \quad \psi_{0}=0 .
$$

Here $\alpha$ is the Lévy exponent and $q$ is a normalization constant. We limit ourselves to the case $1 \leq \alpha \leq 2$, where the averages are finite but the variance not. We formulate the problem by introducing the vector $\mathbf{W} \equiv\left\{W_{i}(t)\right\}$, where $W_{i}$ is the probability for the walker to undergo a scattering event at site $i$ and time $t$. It satisfies a master equation, which, for LFs, writes

$$
\mathbf{W}(t+1)=\mathbf{Q W}(t)+\mathbf{S},
$$

where $\mathbf{S}$ accounts for the particles steadily injected from external resorvoirs (see Fig. 1 for a pictorial representation); $\mathbf{Q}$ is a matrix describing the probability of paths connecting pair of sites. In the simple case of absorbing $\mathrm{BC}$, it is readily seen that $Q_{j i}$ is equal to the probability $\psi_{j-i}$ of a direct flight, as from Eq. (1) (see Fig. 11). In the $\mathrm{LW}$ case, the $\mathbf{W}$ components in the r.h.s. must be estimated at different times (depending on the length of the path followed from $j$ to $i$ ). Since, the stationary solution is the same in both setups, this difference is immaterial, and we thereby refer to LFs, since Eq. (2) is easier to solve iteratively.

In the $\mathrm{LF}$ case, $W_{i}$ is equal to the density $P_{i}$ of particle at site $i$. This is no longer true for the LW, as $P_{i}$ includes those particles that are crossing during a ballistic step. In the simple case of absorbing $\mathrm{BC}$, one can write

$$
P_{i}=W_{i}+\sum_{j=1}^{N} R_{|i-j|} W_{j}+I_{i}
$$

where $R_{i}=\sum_{j>i} \psi_{j}$ and $I_{i}=\sum_{j>i} S_{j}$ account for the particles which, having started their walk respectively inside or outside the domain, transit at the $i$ th site. 


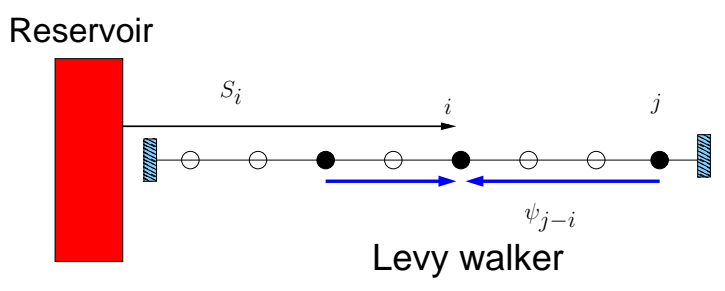

FIG. 1: (Color online) Scheme of the model: an ensemble of Lévy walkers on a finite one-dimensional lattice interacting with one external reservoir that inject particles with a prescribed constant rate $S_{i}$ at each site.

The source term is fixed by assuming that the reservoir is a semi-infinite lattice, homogeneously filled by Lévy walkers of the same type as those residing in the domain. This amounts to defining $S_{m}=s m^{-\alpha}$, where $s$ measures the density of particles and $m$ the distance from the reservoir. It is easy to verify that in the presence of two identical reservoirs at the lattice ends, the density is constant (for any $N$ ), showing that our definition satisfies a kind of "zeroth principle" as it should.

In the nonequilibrium case, it is not necessary to deal with two reservoirs. The linearity of the problem teaches us that it is sufficient to study the case of a single reservoir, that we assume to be in $i=0$ : the effect of, say, a second one on the opposite side can be accounted for by a suitable linear transformation. For values of $N$ large enough, the steady-state density depends on $i$ and $N$ through the combined variable $x=i / N, P_{i}=P(x)$. As seen in Fig. 2 $P$ vanishes for $x \rightarrow 1$ because on that side the absorbing boundary is not accompanied by an incoming flux of particles. Besides, we have also verified that $W_{i} \propto P_{i}$ in agreement with the expectation that LFs and LWs should have by the same stationary properties.

Fractional Laplacian- For a more accurate consistency check, we now compare the solution of our model with that of the stationary Fractional Diffusion Equation (FDE) $D_{x}^{\alpha} P=-\sigma(x)$ on the interval $0 \leq x \leq 1$ (see e.g. Ref. 14] and references therein for a precise definition of the operator $\left.D_{x}^{\alpha}\right)$. The source term $\sigma(x)$ must be chosen so as to describe the effect of the external reservoirs. A condition to be fulfilled is that two identical reservoirs yield a homogeneous state $P(x)=$ const. . A straightforward calculation using the integral definition of the operator [14] shows that this happens for $\sigma(x)=\sigma_{e q}(x) \equiv x^{-\alpha}+(1-x)^{-\alpha}$ (we, henceforth, ignore irrelevant proportionality constants) [22]. It is thus natural to associate $\sigma(x)=x^{-\alpha}$ to the nonequilibrium case with a single source in $x=0$. The numerical solution of the FDE is reported in Fig. 2 (dashed line). It overlaps almost perfectly with the stationary solution of the discrete model, thereby confirming the correctness of our choice. Moreover, this shows that long-ranged sources are needed to reproduce the superdiffusive profiles in the continuum limit.

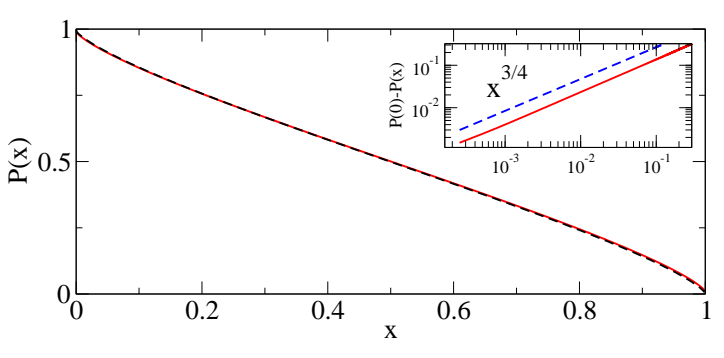

FIG. 2: (Color online) Density profiles as a function of the variable $x=i / N$ for absorbing $\mathrm{BC}$ and $\alpha=3 / 2$. Solid line: solution of the Eq. (2) with $N=4000$. Dashed line: solution of the FDE discretized on a mesh of $M=800$ points; the latter curve is rescaled by a suitable constant. The inset is a magnification of the leftmost region.

Boundary singularity - A distinctive feature of the profile is that it is not analytic at the boundaries. Indeed, the data for $x \rightarrow 0$ are well fitted by

$$
P(x)=P(0)+C x^{\mu}
$$

(the same behavior occurs for $x \rightarrow 1$, as the profiles are symmetric). In view of the similarity with the shape of the liquid surface close to a wall, we metaphorically term $\mu$ as the meniscus exponent. The density drop is akin to the well-known Kapitza resistance, which has been previously discussed for one-dimensional systems [15]. However, the nonanalytic behavior is peculiar of anomalous kinetics, as opposed to the familiar linear shape in standard diffusion. For the above discussed case of absorbing boundaries, we find that $\mu \approx \alpha / 2$. This value is consistent with the singular behavior of the eigenfunctions of the fractional Laplacian [14].

Reflecting boundaries - We now generalize the discrete model, by assuming that any time a particle reaches the boundary, it is reflected with probability $r(0 \leq r \leq 1)$. This extension is of central interest for real problems, e.g. in anomalous diffusion of light [16], but we have to pay the price of a more complicate mathematical treatment to account for multiple reflections. In fact, the transition probability $Q_{j i}$ from the site $j$ to site $i$ is the sum of infinitely many terms that correspond to paths undergoing multiple reflections. By calling $B_{l}$ and $B_{r}$ the left and right boundaries, respectively, and viewing any path as a sequence of symbols that identify the starting and final site, as well as all reflection points, the four families that contribute to $Q_{j i}$ can be encoded as (for $1<j<i<N-1)$ : $j\left(B_{r} B_{l}\right)^{m} i, j B_{l}\left(B_{r} B_{l}\right)^{m} i$, $j B_{r}\left(B_{l} B_{r}\right)^{m} i$, and $j\left(B_{l} B_{r}\right)^{m+1} i$ with $m=0,1, \ldots$ Symmetric relations hold for $j>i$, while special expressions must be invoked for the degenerate cases $(i, j=1, N-1$, $i=j)$. The weight of a path of length $\ell$ is then given by $r^{k} \psi_{\ell}$, where $k$ is the number of reflections $(k=2 m+1$ in the second of the four families). By summing up all contributions for the same pairs of sites, we obtain $Q_{j i}$. A similar treatment must be applied to the source term 
to take into account that each particle entering from either side may be trapped for an arbitrarily long time. Another complication is that for LWs, the expression for $P(x)$ requires including all paths arising from multiple reflections. Notice however, that all such intricacies do not change the rank of the matrix $\mathbf{Q}$.

Unexpectedly, we found that the shape of the profile depends on $r$ for fixed $\alpha$. This is entirely new with respect to normal diffusion, when the profile is linear irrespective of the boundary conditions. The meniscus exponent is a convenient tool to parametrize this functional dependence on $r$. The estimated numerical values of $\mu$ for $\alpha=3 / 2$ are reported in Fig. 3. Once more, we found that $W_{i} \propto P_{i}$ and that, accordingly, both profiles give compatible values of $\mu$ (see symbols in Fig. 3). By assuming a linear dependence of $\mu$ on both $r$, and $\alpha$, we conjecture

$$
\mu=\frac{\alpha}{2}+r\left(\frac{\alpha}{2}-1\right)
$$

This expression is consistent with the $\mu=\alpha / 2$ value found above for $r=0$. Moreover, for $\alpha=2$ (normal diffusion) it yields $\mu=1$, as it should. The discrepancy between formula (5) and the numerical values is everywhere compatible with the finite-size corrections (data has been obtained with $N=8000)$.

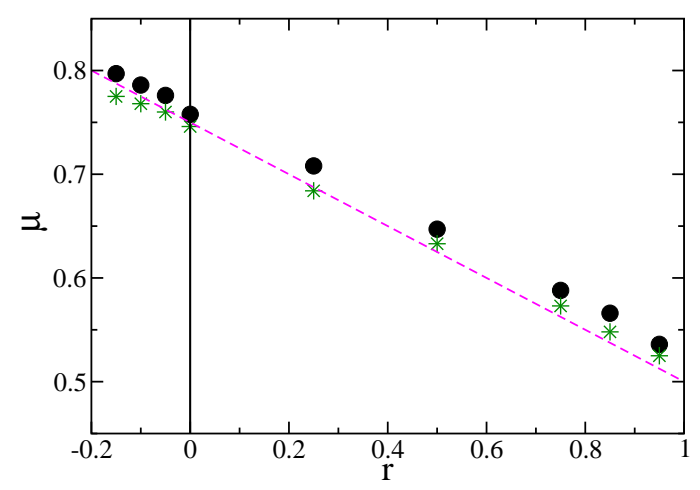

FIG. 3: (Color online) Dependence of the meniscus exponent $\mu$ on the reflection probability $r$ for $\alpha=3 / 2$. Full circles and stars are measures from fitting of $P(x)$ and $W(x)$, respectively (see text). The dashed line is given by the formula (5).

Application to the heat conduction problem- Temperature profiles in one-dimensional systems displaying anomalous energy transport [8] represent a good testing ground of the above approach. In fact, energy perturbations do spread like in a Lévy process [17]. Also, for an infinite chain of harmonic oscillators with energy and momentum conserving noise [18], it was proven that the phonon Boltzmann equation reduces to a FDE with $\alpha=3 / 2$ [19]. We thus expect model (2) to apply to a finite chain of oscillators, (i) coupled with two Langevin heat baths (with a damping constant $\lambda$ ), and (ii) with random collisions that exchange the velocities of neighboring particles with a rate $\gamma$ [20]. This model has the advantage of allowing for an exact solution of the associated Fokker-Planck equation [21].

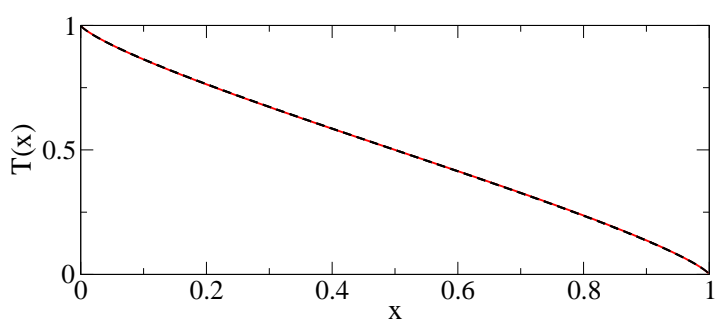

FIG. 4: (Color online) Temperature profile of the oscillator chain with conservative noise with free boundary condition and $\lambda=\gamma=1$ (solid line) and solution of the master equation with reflection coefficient $r=-0.1$ (dashed line).

In Fig. 4 we compare the temperature profile $T(x)$ (suitably shifted and rescaled) of the heat-conduction model with free BC and the solution of our discrete Lévy model with a reflection coefficient $r=-0.1$. Since they are essentially indistinguishable, we can conclude that the Lévy interpretation does not only allow to explain the anomalous scaling of heat conductivity [17], but also the peculiar shape of $T(x)$. The weird (negative) value of $r$ requires a specific discussion. Since $\mathbf{Q}$ is the sum of terms proportional to $r^{k}$ ( $k$ being the number of reflections from the boundaries), even and odd $k$ s yield, for $r<0$, positive and negative contributions, respectively. Thus, we let $\mathbf{Q}=\mathbf{Q}_{e}-\mathbf{Q}_{o}$. The presence of an unphysical negative term can be avoided by introducing two families of walkers $\left(\mathbf{W}^{+}\right.$and $\left.\mathbf{W}^{-}\right)$and interpreting the reflection as a change of family. Under these assumptions, the evolution equations read

$$
\mathbf{W}^{ \pm}(t+1)=\mathbf{Q}_{e} \mathbf{W}^{ \pm}(t)+\mathbf{Q}_{o} \mathbf{W}^{\mp}(t)+\mathbf{S}^{ \pm},
$$

where all terms are positive as they should. Under the further assumption that $\mathbf{S}^{+}=\mathbf{S}$ and $\mathbf{S}^{-}=0$, we can easily see that $\mathbf{W}=\mathbf{W}^{+}-\mathbf{W}^{-}$satisfies the original Eq. (2). In other words, the relevant quantity to look at is the difference between the densities of the two different families. Why it is necessary to invoke the presence of such two families and what is their physical meaning in the context of heat conductivity is an open problem.

In the case of a chain with fixed BC, the temperature profile $T(x)$ can be computed analytically [21] and it is thereby found that $\mu=1 / 2$. By inserting this value in Eq. (5) and recalling that $\alpha=3 / 2$, we find that $r=1$, i.e. the fixed-BC $T(x)$ corresponds to the case of perfectly reflecting barriers. Unfortunately, this (physically reasonable) result could not be tested quantitatively. Indeed, it turns out that finite-size corrections become increasingly important upon increasing $r$, and for $r$ close to 1 , it is practically impossible to achieve convergence to the steady-state.

Local equilibrium- In the context of LWs, we can go beyond the determination of the shape of $P$ and check the 
existence of local equilibrium. To this aim, we decompose $P_{i}$ as $\sum_{n}\left[P_{i}^{R}(n)+P_{i}^{L}(n)\right]$ where $P_{i}^{L, R}(n)$ is the probability that a walker on site $i$ and moving respectively to the left or right will undergo the next scattering event after $n$ time steps. Then, we compare $P_{i}^{L, R}(n)$ with its equilibrium value $P_{e q}(n)$, obtained from the stationary solution of Eq. (2) with two equal sources on both sides $\left[P_{e q}(n)\right.$ is independent of $i$ and of $\left.L, R\right]$. Unsurprisingly, $P_{i}^{L, R}(n) \simeq P_{e q}(n)$ close to the left boundary. Moving away from the reservoir, the densities $P_{i}^{L}(n)$ start to deviate from $P_{e q}(n)$ (see respectively the broken and solid lines in Fig. 5). The $P_{i}^{R}(n)$ (not reported) display similar, though somehow smaller, deviations. Fig. [5] and b refer to the middle of the system and $r=0, r=0.5$, respectively. Deviations decrease upon increasing the system sizes and are larger in the case of absorbing BC, as the particles have less time to "thermalize". Fig. [5] c and d refer to the rightmost site, again for $r=0$ and $r=0.5$. In this case there is no evidence of a convergence towards the equilibrium distribution, but this result is not earthshaking, as the density of particles decreases for increasing $N$. In other words, the deviations concern an asymptotically vanishing fraction of particles.

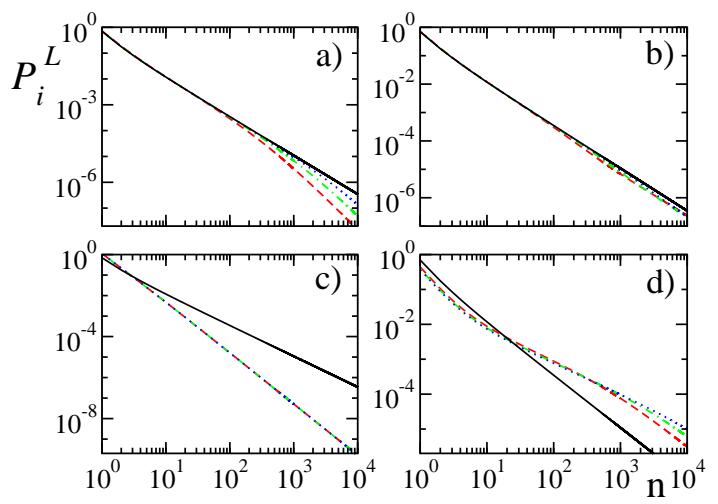

FIG. 5: (Color online) Distributions of left moving particles $P_{i}^{L}(n)$ (broken lines) for $i=N / 2$ and $(\mathrm{a}, \mathrm{b})$ and $i=N(\mathrm{c}, \mathrm{d})$. Dashed, dotted-dashed, and dotted curves correspond to $N=$ 1000, 4000 and 16000, respectively; the solid line is $P_{e q}(n)$; see text for details. All curves are rescaled to span unit area.

Conclusions - In this Letter we have determined the density profiles in the case of superdiffusive motion in an open, finite domain for arbitrary reflectivity $r$. Unexpectedly, their shape depends on both $\alpha$ and $r$ through the meniscus exponent $\mu$, Eq. (5). This is a manifestation of the long-range effects induced by superdiffusive behavior. The prediction of the Lévy model accounts quantitatively for the shape of the temperature profile of an interacting oscillator chain displaying anomalous heat conductivity. It remains to clarify the relationship between the type of $\mathrm{BC}$ in the thermal problem with that in the diffusive one, that appears to be far from trivial.
Another open problem is the rigorous proof of the conjecture (5). We finally hope that the shape can be observed in some optical experiment.

We thank J. Bertolotti for fruitful discussions. This work is part of the PRIN project 2008Y4W3CY.

[1] R. Klages, G. Radons, and I. M. Sokolov, eds., Anomalous Transport: Foundations and Applications (WileyVCH Verlag, Weinheim, 2008).

[2] R. Metzler and J. Klafter, J. Phys A: Math. Gen. 37, R161 (2004).

[3] S. V. Buldyrev, S. Havlin, A. Y. Kazakov, M. G. E. da Luz, E. P. Raposo, H. E. Stanley, and G. M. Viswanathan, Phys. Rev. E 64, 041108 (2001).

[4] P. M. Drysdale and P. A. Robinson, Phys. Rev. E 58, 5382 (1998).

[5] For continuous-time random walks, general $\mathrm{BC}$ have been recently considered in B. P. van Milligen, I. Calvo, and R. Sánchez, J. Phys. A: Math. Theor. 41, 215004 (2008).

[6] S. Denisov, J. Klafter, and M. Urbakh, Phys. Rev. Lett. 91, 194301 (2003).

[7] S. Lepri, C. Mejía-Monasterio, and A. Politi, J. Phys. A: Math. Theor. 43, 065002 (2010).

[8] S. Lepri, R. Livi, and A. Politi, Phys. Rep. 377, 1 (2003).

[9] K. Saito, G. Benenti, and G. Casati, Chem. Phys. 375, 508 (2010).

[10] P. Barthelemy, J. Bertolotti, and D. Wiersma, Nature 453, 495 (2008).

[11] P. Barthelemy, J. Bertolotti, K. Vynck, S. Lepri, and D. S. Wiersma, Phys. Rev. E 82, 011101 (2010).

[12] C. W. Chang, D. Okawa, H. Garcia, A. Majumdar, and A. Zettl, Phys. Rev. Lett. 101, 075903 (2008).

[13] A. Blumen, G. Zumofen, and J. Klafter, Phys. Rev. A 40, 3964 (1989).

[14] A. Zoia, A. Rosso, and M. Kardar, Phys. Rev. E 76, 21116 (2007)

[15] K. Aoki and D. Kusnezov, Phys. Rev. Lett. 86, 4029 (2001).

[16] J. Bertolotti, K. Vynck, and D. S. Wiersma, Phys. Rev. Lett. 105, 163902 (2010).

[17] P. Cipriani, S. Denisov, and A. Politi, Phys. Rev. Lett. 94, 244301 (2005).

[18] G. Basile, C. Bernardin, and S. Olla, Phys. Rev. Lett. 96, 204303 (2006).

[19] M. Jara, T. Komorowski, and S. Olla, The Annals of Applied Probability 19, 2270 (2009).

[20] L. Delfini, S. Lepri, R. Livi, and A. Politi, Phys. Rev. Lett. 101, 120604 (2008).

[21] S. Lepri, C. Mejía-Monasterio, and A. Politi, J. Phys. A: Math. Theor. 42, 025001 (2009).

[22] The FDE is solved by discretizing the fractional Laplacian on a mesh of $M$ equally-spaced sites as given in Ref. [14]. A particular care is required to correctly treat the discretization $\sigma_{i}$ of the source at $i=1, M$. Empirically, we adjusted $\sigma_{1, M}$ to ensure that the equilibrium solution $P=$ const. is accurately reproduced for $\sigma=\sigma_{e q}$. 